\title{
Measuring adaptation following a stroke
}

\author{
Irene Hartigan
}

School of Nursing and Midwifery, UCC

\section{Background}

Stroke is the second commonest cause of mortality worldwide. In Ireland, one in five people will have a stroke at some time in their life. Stroke is the leading cause of longterm disability in Ireland and the incidence of this chronic illness is set to rise due, in part, to the increase in the elderly population. The National Audit of Stroke Care, commissioned by the Irish Heart Foundation and Department of Health and Children in 2006, revealed that stroke services are badly organised in Ireland. Stroke interrupts life, arrests previously-cherished activities and decreases quality of life for survivors and their families. The physical and psychological consequences and changes that stroke survivors suffer can be devastating. The degree of physical disability post stroke can range in effect from moderate to severe. Some of the consequences of stroke, such as mild cognitive impairment, while less apparent, make daily living difficult. Changes in physical, psychological and social roles can affect both one's self-image and the individual in a variety of ways. Little attention is given to the social and psychological aspects of stroke, more commonly termed as psychosocial. Social roles and responsibilities are often not addressed in stroke recovery, yet research has emphasised the importance and contribution of social processes in stroke recovery.

Rehabilitation predominately focuses on minimising stroke-related disability and to enhance active participation in everyday life skills but why is it that in some cases, with the exact same stroke-related deficits, different results are evident? Does their personality influence their response to recovery? Are some people less affected by changes to life post stroke? Are these individuals 'hardy'? The interaction between the personality trait of hardiness and the individuals' response to changes post stroke provided the impetus for this research.

Hardy people believe that they can control or influence events that they experience and anticipate the change as a challenge. Hardiness is coined as a response to stresses associated with chronic illnesses. Hardiness represents integration of specific adaptive tendencies to manage stressors such as those associated with psychological, social and functional processes. Stroke survivors are likely to experience a variety of psychosocial and physical stressors arising from concerns about their illness which has implications for adjustment to their stroke diagnosis and impairments. There is increasing emphasis placed on adjustment to chronic illness rather than recovery as individuals may never achieve complete 
recovery. Evidence suggests that physical or psychosocial gains come mainly from helping patients to adjust to impairments rather than solely by their reduction. Understanding how people navigate the process of adjustment to stroke and applying this understanding to clinical practice will inform rehabilitation services.

\section{Aim}

The aim of this study is to examine the influence of the personality trait hardiness on psychosocial adjustment post stroke.

\section{Methodology}

In order to explore this phenomenon, a quantitative descriptive study will be conducted. Stroke survivors who are not living in institutionalised care will be invited to participate in this study. A questionnaire pack consisting of two reliable and valid scales, as well as a short demographic questionnaire to ascertain characteristics of the study population, will be administered to individuals who have survived a stroke. From the literature reviewed, careful consideration was given to capturing the correct data to measure psychosocial adjustment. Two scales were identified as appropriate in measuring hardiness and psychosocial adjustment. These were the health-related hardiness scale and the psychosocial adjustment to illness scale.

The health-related hardiness scale explores the individual's stress response to the stroke based on the sense of commitment, control and challenge that the individual must possess to adapt to their stroke limitations. The psychosocial adjustment to illness scale is concerned with seven areas of psychosocial adjustment: attitudes towards health, work, family living, sexual relationships, social and leisure activities, anxiety and behavioural interactions with family and friends. No empirical study was found exploring how the personality trait of hardiness influences an individual's psychosocial adjustment to stroke. Measuring the personality trait of hardiness and its relationship to psychosocial adjustment post-stroke may help predict the level of psychosocial adjustment post stroke beyond what could be predicted by other functional measures of outcome.

\section{Findings}

Data collection will be commencing soon. Work to date concentrated on developing the research question and highlighting the gap in stroke research. To this end, it is clear there 
is a paucity of research exploring the personal attribute of hardiness and its influence on psychosocial adjustment to stroke.

\section{Conclusion}

Recovery from stroke is a deeply personal and unique process of changing one's attitudes. While it is crucial for recovery to medically stabilize patients and send them home, more attention should be placed on working with patients and their families, to understand the changes a stroke can cause and how they approach the challenge of rehabilitation. Identity plays an important part in psychosocial adjustment especially for individuals diagnosed with a chronic illness such as stroke. Exploring the personality resource of hardiness may help healthcare professionals uncover knowledge that will help optimise psychosocial adjustment post stroke. Research is needed to identify specific individual adaptive resources that enhance, or at least delay, the onset of stress associated with stroke. Ensuring psychosocial adjustment in stroke survivors can prevent the formation of major psychiatric disorders such as depression. Research advancements in this area have the potential to contribute to higher levels of independent functioning and satisfaction in the later years of life for people.

Thank you to my supervisor Professor Geraldine McCarthy. Irene Hartigan is a College Lecturer and a PhD candidate at the School of Nursing and Midwifery, University College Cork. 